was associated with high disease activity, suggesting that that inflammation might promotes comorbidities. For optimal management of SpA, a systemic screening for comorbidities is essential.

Disclosure of Interests: None declared.

DOI: 10.1136/annrheumdis-2021-eular.2929

\section{POS1001 $\quad$ CLINICAL AND SURROGATE CARDIOVASCULAR RISK ASSESSMENT AND ITS RELATIONSHIP WITH PSORIATIC ARTHRITIS PATHOGENESIS}

N. Barbarroja Puerto ${ }^{1}$, I. Arias de la Rosa ${ }^{1}$, C. Román-Rodriguez ${ }^{2}$, I. Gómez García $^{2}$, C. Perez-Sanchez ${ }^{2}$, C. López-Medina², M. L. Ladehesa Pineda ${ }^{2}$, D. Ruiz ${ }^{2}$, A. M. Patiño-Trives ${ }^{2}$, M. Luque-Tévar² ${ }^{2}$, C. Lopez-Pedrera ${ }^{2}$, A. Escudero Contreras $^{2}$, E. Collantes Estevez ${ }^{2}$, M. D. López Montilla ${ }^{2} .{ }^{1}$ Maimonides Institute for Research in Biomedicine of Cordoba (IMIBIC)/Reina Sofia Hospital/ University of Cordoba, Medicine Department, Cordoba, Spain; ${ }^{2}$ Maimonides Institute for Research in Biomedicine of Cordoba (IMIBIC)/Reina Sofia Hospital/University of Cordoba, Rheumatology Department, Cordoba, Spain

Background: Psoriatic arthritis (PsA) is a chronic inflammatory disease associated with an increased prevalence of cardiovascular (CV) events. Traditional CV risk factors do not account for the increased CV disease mortality in PsA. Inflammation seems to have a key role in the development of this comorbidity, however the specific molecular mechanisms involved are not defined yet.

Objectives: To evaluate clinical CV risk factors and surrogate markers and their relationship with inflammation, disease activity and metabolic comorbidities in PsA patients

Methods: This is a cross-sectional study including 100 PsA patients without CV disease recruited in the routine clinical practice at the Rheumatology Department, Reina Sofia Hospital of Cordoba and 100 age-matched healthy donors (HDs). Different parameters related to the cardiometabolic risk were analyzed. Clinical and analytical parameters were collected: lipid profile (cholesterol, HDL, LDL, TG, ApoA and ApoB), glucose and insulin, body surface area (BSA) affected by psoriasis, number of tender and swollen joints, DAPSA, VAS, CRP and ESR. To measure the persistence of inflammation, CRP levels were recorded retrospectively once, twice, or three times during the 5 years prior to study and at the moment of the study. Increased levels of CRP in at least $50 \%$ of the determinations was considered as persistent inflammation. Plasma and peripheral blood mononuclear cells (PBMCs) were isolated from peripheral blood of patients and HDs. A panel of 92 proteins involved in CV disease and an adipocytokine profile was measured in plasma and PBMCs. In addition, activation of 18 intracellular pathways involved in cell activation was also measured in PBMCs. In vitro experiments in adipocytes treated with serum from PsA patients were also carried out. Results: Traditional CV risk factors including atherogenic risk, insulin resistance (IR), metabolic syndrome, smoking, obesity, arterial hypertension, apolipoprotein B/A risk, type 2 diabetes mellitus and the levels of SCORE were significantly increased in PsA patients. The presence of IR was associated with disease activity markers (DAPSA, ESR and CRP). In fact, the HOMA-IR index was related to the CRP persistence. PsA patients with obesity had significantly increased the number of tender and swollen joints, the levels of DAPSA and CRP. Twenty-eight proteins involved in CV disease and six adipocytokines were significantly elevated in the plasma of PsA patients. Several of these cardiovascular molecules were associated with higher levels of DAPSA (CTSD, GAL3, CD163, FABP4, IL6 and IL1RT2), acute phase reactants (GAL3, TNF $\alpha$, Adiponectin, TNFR1 and IL6), affected body surface area (IL2RA, GAL3, CCL15, TRAP, CSTB, CD163, OPG and CNTN1) and onychopaty (TRAP, VWF, MCP-1, GAL3, LTBR, TFPI, CHI3L1, CTSZ and JAM-A). In addition, the mRNA expression of most of those $28 \mathrm{CV}$ molecules were significantly increased in PBMCs from PSA patients. At intracellular level, the activation of 11 kinases (ERK1/2, AKT, S6 Ribosomal, mTOR, HSP27, Bad, p70 S6 kinase, PRAS40, p53 and caspase-3) involved in insulin signaling, inflammation, cell survival and apoptosis were altered in PBMCs. Finally, serum from PsA patients was able to modify the expression of these molecules in adipocytes. Conclusion: 1) Disease activity and inflammatory burden are closely associated with the presence of metabolic alterations, specifically obesity and IR in patients with PsA. 2) The development of IR is extremely related to the persistence of CRP levels in the previous 5 years. 3 ) Inflammation is closely associated to the adipose tissue dysfunction in PSA and 4) FABP4, CD163 and GAL3 are surrogate $\mathrm{CV}$ markers commonly associated with clinical features of PsA, suggesting the role of these molecules linking CVD and PsA pathogenesis.

Funded by ISCIII (PI17/01316 and RIER RD16/0012/0015) co-funded with FEDER.

Disclosure of Interests: None declared.

DOI: 10.1136/annrheumdis-2021-eular.2990

\section{POS1002 \\ BASELINE CALPROTECTIN AND VISFATIN LEVELS PREDICT RADIOGRAPHIC SPINAL PROGRESSION AFTER 2 YEARS IN ANKYLOSING SPONDYLITIS PATIENTS ON TNF INHIBITOR THERAPY}

J. Rademacher ${ }^{1,2}$, M. Siderius ${ }^{3}$, L. Gellert ${ }^{1}$, F. Wink ${ }^{4}$, M. Verba $^{1}$, F. Maas ${ }^{3}$, L. M. Tietz ${ }^{1}$, D. Poddubnyy ${ }^{1,5}$, A. Spoorenberg ${ }^{3}$, S. Arends ${ }^{3} .{ }^{1}$ Charité - $^{-}$ Universitätsmedizin Berlin, Department of Gastroenterology, Infectiology and Rheumatology, Berlin, Germany; ${ }^{2}$ Berlin Institute of Health, BIH, Berlin, Germany; ${ }^{3}$ University Medical Center Groningen, Department of Rheumatology and Clinical Immunology, Groningen, Netherlands; ${ }^{4}$ Medical Center Leeuwarden, Department of Rheumatology, Leeuwarden, Netherlands ${ }^{5}$ Deutsches Rheuma-Forschungszentrum Berlin (DRFZ), ein Institut der Leibniz-Gemeinschaft, Epidemiology, Berlin, Germany

Background: Radiographic spinal progression determinates functional status and mobility in ankylosing spondylitis (AS) ${ }^{1}$.

Objectives: To analyse whether biomarker of inflammation, bone turnover and adipokines at baseline or their change after 3 months or 2 years can predict spinal radiographic progression after 2 years in AS patients treated with TNF- $\alpha$ inhibitors (TNFi).

Methods: Consecutive AS patients from the Groningen Leeuwarden Axial Spondyloarthritis (GLAS) cohort ${ }^{2}$ starting TNFi between 2004 and 2012 were included. The following serum biomarkers were measured at baseline, 3 months and 2 years of follow-up with ELISA:

- Markers of inflammation: calprotectin, matrix metalloproteinase-3 (MMP-3), vascular endothelial growth factor (VEGF)

- Markers of bone turnover: bone-specific alkaline phosphatase (BALP), serum C-terminal telopeptide (sCTX), osteocalcin (OC), osteoprotegerin (OPG), procollagen typ I and II N-terminal propeptide (PINP; PIINP), sclerostin.

Adipokines: high molecular weight (HMW) adiponectin, leptin, visfatin Two independent readers assessed spinal radiographs at baseline and 2 years of follow-up according to the modified Stoke Ankylosing Spondylitis Spine Score (mSASSS). Radiographic spinal progression was defined as mSASSS change $\geq 2$ units or the formation of $\geq 1$ new syndesmophyte over 2 years. Logistic regression was performed to examine the association between biomarker values at baseline, their change after 3 months and 2 years and radiographic spinal progression. Multivariable models for each biomarker were adjusted for mSASSS or syndesmophytes at baseline, elevated CRP ( $\geq 5 \mathrm{mg} / \mathrm{l}$ ), smoking status, male gender, symptom duration, $\mathrm{BMI}$, and baseline biomarker level (the latter only in models with biomarker change).

Results: Of the 137 included AS patients, $72 \%$ were male, $79 \%$ HLAB27+; mean age at baseline was 42 years (SD 10.8), ASDAScrp $3.8(0.8)$ and mSASSS 10.6 (16.1). After 2 years of follow-up, 33\% showed mSASSS change $\geq 2$ units and $24 \%$ had developed $\geq 1$ new syndesmophyte. Serum levels of biomarkers of inflammation and bone formation showed significant changes under TNFi therapy, whereas adipokine levels were not altered from baseline (Figure 1).

Univariable logistic regression revealed a significant association of baseline visfatin (odds ratio OR [95\% confidence interval] 1.106 [1.007-1.215]) and sclerostin serum levels (OR 1.006 [1.001-1.011]) with mSASSS progression after 2 years. Baseline sclerostin levels were also associated with syndesmophyte progression (OR 1.007 [1.001-1.013]). In multivariable logistic analysis, only baseline visfatin level remained significantly associated (OR 1.465 [1.137-1.889]) with mSASSS progression. Furthermore, baseline calprotectin showed a positive association with both, mSASSS (OR 1.195 [1.055-1.355]) and syndesmophyte progression (OR 1.107 [1.001-1.225]) when adjusting for known risk factors for radiographic progression.

Univariable logistic regression showed that change of sclerostin after 3 months was associated with syndesmophytes progression (OR 1.007 [1.000-1.015), change of PINP level after 2 years was associated with mSASSS progression (OR 1.027 [1.003-1.052]) and change of visfatin after 2 years was associated with both measures of radiographic progression - mSASSS (OR 1.108 [1.004-1.224]) and syndesmophyte formation (OR 1.115; [1.002-1.24]). However, those associations were lost in multivariable analysis.

Conclusion: Independent of known risk factors, baseline calprotectin and visfatin levels were associated with radiographic spinal progression after 2 years of TNFi. Although biomarkers of inflammation and bone formation showed significant changes under TNFi therapy, these changes were not significantly related to radiographic spinal progression in our cohort of AS patients.

\section{REFERENCES:}

[1] Poddubnyy et al 2018

[2] Maas et al 2019 
A

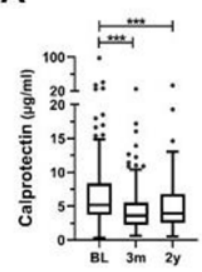

D

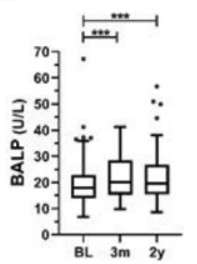

G

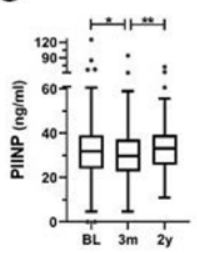

J

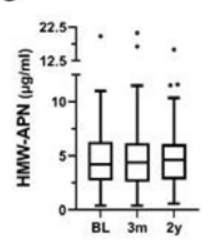

B

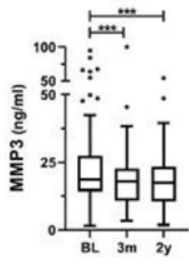

E

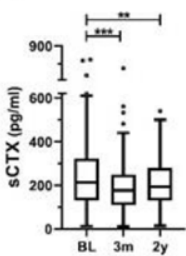

H

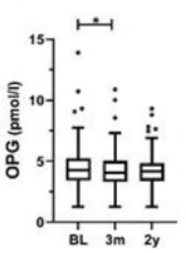

K

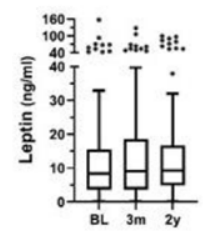

C

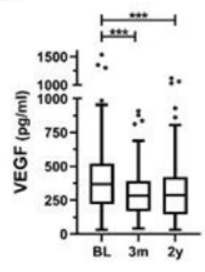

F

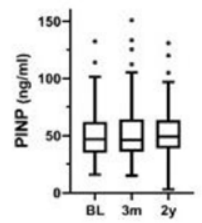

I

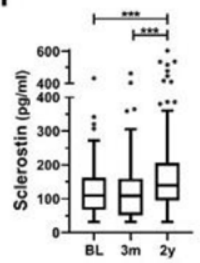

L

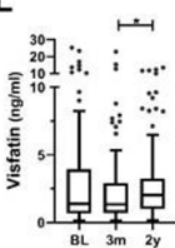

Figure. Levels of markers of inflammation (A-C), bone turnover markers (D-I) and adipokines (J-L) under treatment with TNF inhibitors: serum levels at baseline (BL; before start of TNFi), after 3 months $(3 \mathrm{~m})$ and 2 years $(2 \mathrm{y})$ of treatment. Wilcoxon Signed Rank Test: ${ }^{\star} \mathrm{p}$ value $<0.05 ;{ }^{\star \star} \mathrm{p}$ value $<0.01 ;{ }^{\star \star \star} \mathrm{p}$ value $<0.001$

Acknowledgements: Dr. Judith Rademacher is participant in the BIH-Charité Clinician Scientist Program funded by the Charité -Universitätsmedizin Berlin and the Berlin Institute of Health.

Disclosure of Interests: Judith Rademacher: None declared, Mark Siderius: None declared, Laura Gellert: None declared, Freke Wink Consultant of: AbbVie, Maryna Verba: None declared, Fiona Maas: None declared, Lorraine M Tietz: None declared, Denis Poddubnyy: None declared, Anneke Spoorenberg Consultant of: Abbvie, Pfizer, MSD, UCB, Lilly and Novartis, Grant/research support from: Abbvie, Pfizer, UCB, Novartis, Suzanne Arends Grant/research support from: Pfizer. DOI: 10.1136/annrheumdis-2021-eular.3059

\section{POS1003 CATASTROPHIZING IN PATIENTS WITH SPONDYLOARTHRITIS}

B. Coste ${ }^{1}$, C. Traverson ${ }^{1}$, E. Filhol ${ }^{1,2}$, C. Lukas ${ }^{2,3}$, S. Laurent-Chabalier ${ }^{4}$, J. Morel ${ }^{3,5}$, B. Combe ${ }^{3,5}$, C. Daien ${ }^{3,5}$, C. Hua ${ }^{1,2}$, C. Gaujoux-Viala ${ }^{1,2}{ }^{1} N$ îmes University Hospital, Rheumatology, Nîmes, France; ${ }^{2}$ University of Montpellier, IDESP, UMR-INSERM, Montpellier, France; ${ }^{3} \mathrm{CHU}$ Lapeyronie, Rheumatology, Montpellier, France; ${ }^{4}$ Nîmes University Hospital, BESPIM, Nîmes, France; ${ }^{5}$ University of Montpellier, -, Montpellier, France

Background: Catastrophizing is a negative cognitivo-affective response to an anxiety-provoking stimulus, especially anticipated or actual pain. It can be assessed quickly using a validated questionnaire: the Pain Catastrophizing Scale (PCS) ${ }^{1}$. Catastrophizing plays a role in maintaining chronic pain and is associated with several pain-related outcomes in osteoarthritis and low back pain. There is a lack of knowledge about catastrophizing in axial spondyloarthritis (AS) with only one study ${ }^{2}$ so far.

Objectives: To assess the prevalence of catastrophizing and associated factors in spondyloarthritis.

Methods: We performed an observational, prospective, bi-centric study. All patients aged 18 or over with AS fulfilling the 2009 Assessment in Spondyloarthritis International Society (ASAS) criteria were consecutively included. Sociodemographic data, information on the disease and its treatments were collected as well as questionnaires regarding disease activity (BASDAI), function (HAQ, BASFI), quality of life (SF12,

EQ5D), anxiety and depression (HADS, GAD7), fibromyalgia (FiRST), insomnia (ISI) and catastrophizing scores (PCS). Statistical analysis included a samples t-test, oneway variance analysis, Spearman's correlation coefficient, the $\mathrm{Chi}^{2}$ test, Fisher's exact test, the Wilcoxon test, multivariate linear regression (considering catastrophizing as a continuous variable) and multivariate logistics regression (considering catastrophizing as a categorical variable: PCS $\geq 20=$ high level catastrophizing).

Results: From September 2019 to March 2020, 168 AS patients were included: 48.5\% were women, the median age was 48.5 years and 100 patients $(60.2 \%)$ were professionally active. Almost all patients (95.8\%) had a disease lasting for more than 2 years; $110(72 \%)$ were HLA-B27+; 84 (50\%) had MRI sacroilitis and 62 (37.6\%) radiographic sacroiliitis. In all, $166(98.8 \%)$ had axial involvement, $99(58.9 \%)$ had peripheral involvement and $44(26.2 \%)$ had enthesitic involvement. The median BASDAI score was 6.30 [Q1-Q3 4.65-6.30].

The prevalence of a PCS score $\geq 20$ was $45.5 \%$ [38.0;53.0]. The median PCS score was 18 [7-27]. In multivariate logistics regression, high-level catastrophizing was significantly associated with the HADS anxiety score (OR=1.54 [1.22-2.0]), HADS depression score $(\mathrm{OR}=1.25$ [1.10-1.43]) and disease activity (BASDAI OR=1.14 [1.011.26]). In multivariate linear regression, catastrophizing was also significantly associated with anxiety $(p<0.0001)$, depression $(p<0.0001)$ and disease activity $(p=0.0008)$. Conclusion: Almost half the patients with AS were high catastrophizers. Catastrophizing is linked to anxiety, depression, and disease activity. It may be interesting to detect catastrophizing in order to improve the management of our patients.

\section{REFERENCES:}

[1] Sullivan MJL. et al. Psychological Assessment. 1995;7(4):524-32

[2] Penhoat M. et al. Joint Bone Spine. 2014;81(3):235-9

Disclosure of Interests: None declared.

DOI: 10.1136/annrheumdis-2021-eular.3171

\section{\begin{tabular}{l}
\hline POS1004 \\
BOTH SPONDYLOARTHRITIS AND PSORIATIC \\
ARTHRITIS PATIENTS HAVE STRONG FAMILY
\end{tabular} HISTORIES}

G. K. Yardımcı ${ }^{1}$, B. Farisoğulları ${ }^{1}$, E. C. Bolek ${ }^{1}$, E. Bilgin ${ }^{1}$, E. Duran ${ }^{1}$, G. Ayan ${ }^{1}$, Z. Özsoy ${ }^{1}$, G. Sandal Uzun ${ }^{1}$, L. Kılıç ${ }^{1}$, A. Akdoğan ${ }^{1}$, O. Karadag ${ }^{1}$, Ş. A. Bilgen ${ }^{1}$, A. İ. Ertenli ${ }^{1}$, U. Kalyoncu ${ }^{1}$, S. Kiraz ${ }^{1} .{ }^{1}$ Faculty of Medicine; Hacettepe University, Rheumatology, Ankara, Turkey

Background: Family history is one of the hallmarks of spondyloarthritis (SpA) and psoriatic arthritis (PsA) [1, 2]. Some patients have a strong family history that more than 2 relatives have spondyloarthritis related diseases. The effects of strong family history on SpA features were not known very well.

Objectives: The aim of this study is to evaluate the effects of family history in SpA and PsA patients.

Methods: HUR-BIO (Hacettepe University Biologic Registry) is a prospective, single center database of biological treatments since 2005, and to date $3071 \mathrm{SpA}$ and 526 PsA patients have been recorded. Demographic, clinical characteristics, disease activity parameters, a detailed family history of SpA and SpA features (presence of SpA including PsA, psoriasis, inflammatory bowel disease and uveitis) and laboratory data before anti-TNF treatments of the patients were noted.

Results: 2807 SpA (53.6\% male) and 506 PsA (31.4\% male) patients' family history were available and analysed. A positive family history was noted in $27.6 \%$ of the SpA and $31.0 \%$ of the PsA patients (ns). $7.4 \%$ of the SpA patients and $8.9 \%$ of the PsA patients had family history in more than one relative (Table 1). In SpA patients with a family history, uveitis was more frequent than patients without (14.4\% vs $10.6 \%, p=0.006)$. Except for a higher male predominance and uveitis $(53 \%$ vs $32 \%$ $\mathrm{p}=0.006$ and $9 \%$ vs $2 \% \mathrm{p}=0.003$ respectively) in patients with $\geq 2$ relatives with $\mathrm{SpA}$ features, there were no differences in PsA patients regarding family history. The presence of family history and HLA-B27 (63.7\% vs 37.6\%, p<0.001) positivity were associated in SpA patients but not in PsA patients (31.2\% vs $20.0, p=0.13$ ).

Conclusion: Family history was present in about one third of the patients of PsA and $\mathrm{SpA}$. It is not uncommon for two or more family members to have a SpA feature. Presence of family history may be associated with some clinical conditions, such as uveitis. REFERENCES:

[1]Solmaz, D., et al., Impact of Having Family History of Psoriasis or Psoriatic Arthritis on Psoriatic Disease. Arthritis Care Res (Hoboken), 2020. 72(1): p. 63-68.

[2] Zurita Prada, P.A., et al., Influence of smoking and obesity on treatment response in patients with axial spondyloarthritis: a systematic literature review. Clin Rheumatol, 2020.

Table 1. Family history in PsA and SpA patients

\begin{tabular}{|c|c|c|}
\hline & PsA $(n=506)$ & SpA $(n=2807)$ \\
\hline $\begin{array}{l}\geq 1 \text { family history, } n(\%) \\
\geq 1 \text { first-degree relative, } n(\%) \\
\geq 2 \text { first-degree relatives, } n(\%) \\
\geq 2 \text { relatives (both first- and second-degree), } n(\%) \\
\text { Family history }\end{array}$ & $\begin{array}{l}157(31.0) \\
114(22.5) \\
21(4.2) \\
45(8.9)\end{array}$ & $\begin{array}{l}774(27.6) \\
489(17.4) \\
77(2.7) \\
208(7.4)\end{array}$ \\
\hline $\begin{array}{l}>\text { Psoriasis, } \mathbf{n}(\%) \\
>\text { Psoriatic arthritis, } \mathbf{n}(\%) \\
>\text { Spondyloarthritis, } \mathbf{n}(\%) \\
\text { > Inflammatory bowel disease, } \mathrm{n}(\%) \\
\text { > Uveitis, } \mathrm{n}(\%)\end{array}$ & $\begin{array}{l}120(23.7) \\
14(2.8) \\
38(7.5) \\
1(0.2) \\
0\end{array}$ & $\begin{array}{l}155(5.5) \\
9(0.3) \\
643(22.9) \\
10(0.4) \\
2(0.1)\end{array}$ \\
\hline
\end{tabular}

